\title{
Construction of a family of non-stationary biorthogonal wavelets
}

\author{
Baoxing Zhang ${ }^{1 *}$, Hongchan Zheng ${ }^{1}$, Jie Zhou ${ }^{1}$ and Lulu Pan ${ }^{1}$
}

"Correspondence:

baoxingzhang@yeah.net

'Department of Applied

Mathematics, Northwestern

Polytechnical University, Xi'an,

P.R. China

\section{囪 Springer}

\begin{abstract}
The family of exponential pseudo-splines is the non-stationary counterpart of the pseudo-splines and includes the exponential B-spline functions as special members. Among the family of the exponential pseudo-splines, there also exists the subclass consisting of interpolatory cardinal functions, which can be obtained as the limits of the exponentials reproducing subdivision. In this paper, we mainly focus on this subclass of exponential pseudo-splines and propose their dual refinable functions with explicit form of symbols. Based on this result, we obtain the corresponding biorthogonal wavelets using the non-stationary Multiresolution Analysis (MRA). We verify the stability of the refinable and wavelet functions and show that both of them have exponential vanishing moments, a generalization of the usual vanishing moments. Thus, these refinable and wavelet functions can form a non-stationary generalization of the Coifman biorthogonal wavelet systems constructed using the masks of the $D-D$ interpolatory subdivision.
\end{abstract}

Keywords: Pseudo-splines; Exponential pseudo-splines; Vanishing moments; Coifman biorthogonal wavelets

\section{Introduction}

During the last decades, biorthogonal wavelets have been proved to be very successful tools in engineering and applied mathematics. Their properties like symmetry, vanishing moments, regularity and short support are required in different applications such as image compression and signal processing. Due to such properties, there has been continuous work on the construction and application of the biorthogonal wavelets; see for example [15]. In [1], Cohen et al. gave biorthogonal bases derived from compactly supported duals of B-splines. Wei et al. [2] constructed Coifman biorthogonal wavelet systems with vanishing moments equally distributed for refinable and wavelet functions. Kim et al. [3] presented a new family of biorthogonal wavelet systems based on a class of quasi-interpolatory subdivision [6]. More generally, based on the pseudo-splines [7-9], Dong and Shen [4] constructed biorthogonal wavelets with prescribed regularity. Zhou and Zheng [5] obtained biorthgonal wavelets from the smoothed pseudo-splines [10].

Although the above biorthogonal wavelets own excellent properties, they are scaleindependent and lack some flexibility. Non-stationary biorthogonal wavelets, however, are scale-dependent and thus can be more flexible than the stationary ones. This kind of biorthogonal wavelets can be tuned to wider classes of signals and can also ensure good approximation and sparsity properties [11]. For such biorthogonal wavelets, Vonesch et

(c) The Author(s) 2019. This article is distributed under the terms of the Creative Commons Attribution 4.0 International License (http://creativecommons.org/licenses/by/4.0/), which permits unrestricted use, distribution, and reproduction in any medium, provided you give appropriate credit to the original author(s) and the source, provide a link to the Creative Commons license, and indicate if changes were made. 
al. [11] constructed generalized biorthogonal wavelets generating exponential polynomials. The corresponding wavelet functions have exponential vanishing moments, a generalization of the usual vanishing moments [11]. Lee and Yoon [12] analyzed the compactly supported non-stationary biorthogonal wavelets in [11]. Note that these obtained nonstationary biorthogonal wavelets are mainly based on the exponential B-splines. In fact, Conti et al. [13] presented the family of exponential pseudo-splines, the non-stationary generalization of the pseudo-splines [13], and the exponential B-splines form a special subclass of the exponential pseudo-splines. These exponential pseudo-splines can actually meet various demands for balancing approximation order, regularity, support size, interpolation and reproduction capabilities.

Apart from the exponential B-splines, the family of exponential pseudo-splines also contains another subclass consisting of interpolatory cardinal functions, which can be seen as the non-stationary counterparts of the pseudo-splines of type II with order $(m, m-1)$ (see [13]). In fact, the generalized Daubchies wavelets obtained by Dyn et al. [14] can be seen as based on such exponential pseudo-splines. In this paper, inspired by the abovementioned work, we mainly focus on this subclass of exponential pseudo-splines and construct a family of non-stationary biorthogonal wavelets. In fact, to obtain the desired biorthogonal wavelets, we first derive the explicit form of the symbols of the dual refinable functions for this subclass of exponential pseudo-splines. Then the corresponding biorthogonal wavelets can be obtained by the non-stationary MRAs. For the new refinable and wavelet functions, we show their stability and that both of them have the exponential vanishing moments. As a result, they can be seen as the generalization of the stationary Coifman biorthogonal wavelets constructed using the masks of the D-D interpolatory subdivision [3]. We point out that, in a similar way, the biorthogonal wavelets based on other exponential pseudo-splines can also be obtained. But the explicit form of the symbols of the dual refinable functions is difficult to derive in general.

The rest of this paper is organized as follows. In Sect. 2, we review the exponential pseudo-splines and some related definitions and results. In Sect. 3, we derive the dual refinable functions of the subclass of the exponential pseudo-splines consisting of interpolatory cardinal functions and investigate their stability. Based on this result, in Sect. 4, we derive the corresponding biorthogonal wavelets and show their exponential vanishing moments. An example of these non-stationary biorthogonal wavelets is also given in this section. Section 5 concludes this paper.

\section{Preliminaries}

In this section, we review the exponential pseudo-splines and some related knowledge. As the exponential pseudo-splines are closely connected with non-stationary subdivision, we first give a brief introduction to such kind of subdivision schemes.

Let $l_{0}(\mathbb{Z})$ denote the linear space of real sequences with finite length. Given an initial data sequence $\boldsymbol{q}^{0}=\left\{q_{n}^{0} \in \mathbb{R}: n \in \mathbb{Z}\right\} \in l_{0}(\mathbb{Z})$, a binary non-stationary subdivision scheme is defined through

$$
\boldsymbol{q}_{i}^{k+1}=\left(S_{\boldsymbol{a}^{k}} \boldsymbol{q}^{k}\right)_{i}:=\sum_{j \in \mathbb{Z}} a_{i-2 j}^{k} q_{j}^{k}, \quad j \in \mathbb{Z}, k \geq 0
$$


where the sequence $\boldsymbol{a}^{k} \in l_{0}(\mathbb{Z})$ is termed the $k$-level mask and we denote this subdivision by $\left\{S_{\boldsymbol{a}^{k}}\right\}_{k \geq 0}$. The symbol associated with the mask $\boldsymbol{a}^{k}$ is the Laurent polynomial

$$
a^{k}(z)=\sum_{i \in \mathbb{Z}} a_{i}^{k} z^{i}, \quad z \in \mathbb{C} \backslash\{0\} .
$$

If the mask $\boldsymbol{a}^{k}$ is independent of $k$, the subdivision $\left\{S_{\boldsymbol{a}^{k}}\right\}_{k \geq 0}$ becomes a stationary one and we denote it by $S_{\boldsymbol{a}}$.

The scheme $\left\{S_{\boldsymbol{a}^{k}}\right\}_{k \geq 0}$ becomes an interpolatory subdivision if $\boldsymbol{a}^{k}$ satisfies

$$
a_{2 i}^{k}=\delta_{i, 0}, \quad i \in \mathbb{Z}, k \geq 0,
$$

where $\delta_{i, 0}=1$ if $i=0$ and zero otherwise. In terms of symbols, (1) is equivalent to

$$
a^{k}(z)+a^{k}(-z)=2, \quad z \in \mathbb{C} \backslash\{0\}, k \geq 0 .
$$

Let $\delta:=\left\{\delta_{i, 0}, i \in \mathbb{Z}\right\}$. Following [15], we give the following definition on the $C^{v}$ convergence of the scheme $\left\{S_{\boldsymbol{a}^{k}}\right\}_{k \geq 0}$ with $v \geq 0$.

Definition 1 ([15]) The subdivision $\left\{S_{\boldsymbol{a}^{k}}\right\}_{k \geq 0}$ is $C^{\nu}$ if, for the initial data $\boldsymbol{q}^{0}:=\boldsymbol{\delta}$, there exists a function $f_{\mathbf{q}^{0}} \in C^{v}, f_{\boldsymbol{q}^{0}} \neq \equiv 0$, such that

$$
\lim _{k \rightarrow \infty} \sup _{i \in \mathbb{Z}}\left|f_{\boldsymbol{q}^{0}}\left(2^{-k} i\right)-\boldsymbol{q}_{i}^{k}\right|=0 \text {. }
$$

For the convergent non-stationary subdivision schemes, one of the important properties is the generation/reproduction of exponential polynomials. Here, generation means producing a specific type of limit functions by a subdivision and reproduction refers to the capability of a subdivision to reproduce in the limit exactly the same function from which the data is sampled [13].

Now let $\imath$ denote the imaginary unit satisfying $\iota^{2}=-1$. We first recall the space of exponential polynomials.

Definition 2 ([13]) For $n \in \mathbb{Z}_{+}$, let $\Gamma:=\left\{\left(\theta_{1}, \tau_{1}\right), \ldots,\left(\theta_{n}, \tau_{n}\right)\right\}$ with $\theta_{i} \in \mathbb{R} \cup \imath \mathbb{R}, \theta_{i} \neq \theta_{j}$ if $i \neq j$ and $\tau_{i} \in \mathbb{Z}_{+}$denoting the multiplicity of $\theta_{i}, i=1, \ldots, n$. The space of exponential polynomials $E P_{\Gamma}$ is defined to be

$$
E P_{\Gamma}:=\operatorname{span}\left\{x^{r_{i}} e^{\theta_{i} x}, r_{i}=0, \ldots, \tau_{i}-1, i=1, \ldots, n\right\}
$$

In this paper, for the set $\Gamma$ defined in Definition 2, we let $\sum_{i=1}^{n} \tau_{i}=2 m$ with $m \in \mathbb{Z}_{+}$and set $\Gamma$ to be a symmetric set of the form

$$
\Gamma=\left\{\left(\theta_{r}, \tau_{r}\right),\left(-\theta_{r}, \tau_{r}\right)\right\}_{r=0, \ldots, s-1}, \quad s \in \mathbb{Z}_{+} .
$$

The following result gives conditions on $a^{k}(z)$ for $\left\{S_{a^{k}}\right\}_{k \geq 0}$ to generate/ reproduce $E P_{\Gamma}$.

Theorem 1 ([16]) The scheme $\left\{S_{\boldsymbol{a}^{k}}\right\}_{k \geq 0}$ generates $E P_{\Gamma}$ if $a^{k}(z)$ satisfies

$$
\frac{d^{r} a^{k}\left(-e^{-\theta_{i} 2^{-k-1}}\right)}{d z^{r}}=0, \quad r=0, \ldots, \tau_{i}-1,\left(\theta_{i}, \tau_{i}\right) \in \Gamma .
$$


If, in addition, $a^{k}(z)$ satisfies

$$
\frac{d^{r} a^{k}\left(e^{-\theta_{i} 2^{-k-1}}\right)}{d z^{r}}=2 \delta_{r}, \quad r=0, \ldots, \tau_{i}-1,\left(\theta_{i}, \tau_{i}\right) \in \Gamma,
$$

besides (4), then the scheme $\left\{S_{\boldsymbol{a}^{k}}\right\}_{k \geq 0}$ reproduces $E P_{\Gamma}$.

Given the initial data sequence $\delta$, when the scheme $\left\{S_{\boldsymbol{a}^{k}}\right\}_{k \geq 0}$ is convergent, we can obtain the basic limit function $\phi^{k}$, which is generated by

$$
\phi^{k}:=\lim _{l \rightarrow \infty} S_{\boldsymbol{a}^{k+l}} \cdots S_{\boldsymbol{a}^{k+1}} S_{\boldsymbol{a}^{k}} \boldsymbol{\delta}, \quad k \geq 0 .
$$

The basic limit functions $\phi^{k}$ are compactly supported and mutually refinable, i.e. they satisfy the non-stationary refinement equations

$$
\phi^{k}=\sum_{j \in \mathbb{Z}} a_{j}^{k} \phi^{k+1}(2 \cdot-j), \quad k \geq 0
$$

For $\phi^{k}, \boldsymbol{a}^{k}$ and $a^{k}(z)$ are also termed its mask and symbol, respectively.

The exponential pseudo-splines actually form a large family of such non-stationary refinable functions. Starting from the stationary pseudo-splines, they can be seen as derived by replacing polynomial reproduction/generation by exponential polynomial reproduction/generation [13]. The exponential pseudo-splines can be obtained as the basic limit functions of non-stationary subdivision with symbols derived as a 'correction' of the exponential B-spline symbols [13].

Specifically speaking, in this paper, with the set $\Gamma$ in (3), let

$$
b^{k}(z)=2 \sum_{r=0}^{s-1}\left(\frac{e^{\frac{\theta_{r}}{k+1}} z+1}{2}\right)^{\tau_{r}}\left(\frac{e^{\frac{-\theta_{r}}{2^{k+1}}} z+1}{2}\right)^{\tau_{r}} z^{-m}
$$

denote the $k$-level symbol of the exponential B-spline scheme generating $E P_{\Gamma}$. Similar to the set $\Gamma$, we let $\tilde{\Gamma}=\left\{\left(\theta_{i}, \tilde{\tau}_{i}\right)\right\}_{i=1, \ldots, \tilde{n}}$ with $\tilde{\tau}_{i} \leq \tau_{i}$ for $i=1, \ldots, \tilde{n}$ and $\tilde{n} \leq n$. We also set $\tilde{\Gamma}$ to be a symmetric set as $\Gamma$. From [13], we can find the unique Laurent polynomial $c^{k}(z)$ with the lowest possible degree such that the subdivision with the symbol $a^{k}(z)=b^{k}(z) c^{k}(z)$ generates $E P_{\Gamma}$ and reproduces $E P_{\tilde{\Gamma}}$. Here, $E P_{\tilde{\Gamma}}$ is a subspace of $E P_{\Gamma}$ identified by $\tilde{\Gamma}$. Then the exponential pseudo-spline with the symbol $a^{k}(z)$ can be found, which is obtained as the basic limit function of this subdivision (also called the exponential pseudo-spline subdivision) [13]. In particular, when $E P_{\tilde{\Gamma}}=E P_{\Gamma}, a^{k}(z)$ satisfies (2) and this exponential pseudospline becomes the non-stationary interpolatory cardinal function whose limit stationary counterpart is the pseudo-splines of type II with order $(m, m-1)[13,17]$. In this way, as a large family of non-stationary refinable functions, these exponential pseudo-splines provide flexibility in wavelet constructions and filter designs. We point out that the biorthogonal wavelets in this paper will be based on the exponential pseudo-splines in the case of $E P_{\tilde{\Gamma}}=E P_{\Gamma}$.

The existence of the non-stationary refinable functions is the key ingredient for the non-stationary multiresolution analysis (MRA). Let $\left(V^{k}\right)_{k \geq 0}$ be a sequence of subspaces 
in $L^{2}(\mathbb{R})$, with

$$
V^{k}=\overline{\operatorname{span}\left\{\phi_{i}^{k}:=2^{\frac{k}{2}} \phi^{k}\left(2^{\frac{k}{2}} \cdot-i\right)\right\}} .
$$

This sequence of subspaces forms a non-stationary MRA in $L^{2}(\mathbb{R})$ if [18]

(i). $V^{k} \subset V^{k+1}$ for $k \in \mathbb{Z}$;

(ii). $\bigcup_{k \in \mathbb{Z}} V^{k}$ is dense in $L^{2}(\mathbb{R})$;

(iii). for each $k \in \mathbb{Z}$, the set $\left\{\phi_{j}^{k}: j \in \mathbb{Z}\right\}$ forms a Riesz basis of $V^{k}$.

A refinable function $\tilde{\phi}^{k} \in L^{2}(\mathbb{R})$ is the dual refinable function of $\phi^{k}$ if

$$
\left\langle\phi^{k}, \tilde{\phi}^{k}(\cdot-n)\right\rangle=\delta_{n, 0}, \quad k \geq 0, n \in \mathbb{Z}
$$

With $\phi^{k}$ and $\tilde{\phi}^{k}$, a pair of non-stationary MRAs $\left(V_{k}\right)_{k \geq 0}$ and $\left(\tilde{V}_{k}\right)_{k \geq 0}$ can be obtained. Then the general aim of the biorthogonal wavelets consists of finding the complement spaces $W_{k}$ and $\tilde{W}_{k}$ of $V_{k}$ and $\tilde{V}_{k}$, respectively, such that

$$
V_{k+1}=V_{k} \oplus W_{k}, \quad \tilde{V}_{k+1}=\tilde{V}_{k} \oplus \tilde{W}_{k}, \quad W_{k} \perp \tilde{V}_{k}, \quad \tilde{W}_{k} \perp V_{k} .
$$

Thus, given the refinable function $\phi^{k}$, the first step of the construction of the nonstationary biorthogonal wavelets is to obtain the dual refinable function $\tilde{\phi}^{k}$.

Note that the exponential pseudo-splines in the subclass consisting of interpolatory cardinal refinable functions can be seen as the basic limit functions of the interpolatory exponentials reproducing subdivision and are linearly independent. Here, the concept of linear independence is related to the weaker concept of stability. It is known that, for any fixed $k \geq 0$, the set $\left\{\phi^{k}(\cdot-i): i \in \mathbb{Z}\right\}$ is stable if and only if there exist constants $A^{k}, B^{k}>0$ such that

$$
A^{k} \leq\left[\hat{\phi}^{k}, \hat{\phi}^{k}\right] \leq B^{k}
$$

where the bracket product $[\cdot, \cdot]$ is defined as

$$
[f, g](\xi)=\sum_{n \in \mathbb{Z}} f(\xi+2 n \pi) \overline{g(\xi+2 n \pi)}, \quad f, g \in L^{2}(\mathbb{R})
$$

and $\hat{\phi}^{k}$ denotes the Fourier transform of $\phi^{k}$. The set $\left\{\phi^{k}(\cdot-i): i \in \mathbb{Z}\right\}$ forms a Riesz basis if (8) holds. Besides, if the set $\left\{\phi^{k}(\cdot-i): i \in \mathbb{Z}\right\}$ is linearly independent, $\phi^{k}(x)$ is then stable [19].

\section{The dual refinable functions}

In this section, we derive the dual refinable functions of the exponential pseudo-splines in the case of $E P_{\tilde{\Gamma}}=E P_{\Gamma}$, which are interpolatory cardinal refinable functions.

\subsection{Construction of the dual refinable functions}

Given the set $\Gamma$ in (3), let $\phi^{k}$ belongs to the subclass of exponential pseudo-splines consisting of the interpolatory refinable functions. From Sect. 2, it is generated as the basic limit function of the subdivision generating and reproducing the same space, i.e. $E P_{\Gamma}$. For 
a refinable function $\tilde{\phi}^{k}$ with the symbol $\tilde{a}^{k}(z)$, a necessary condition for $\phi^{k}$ and $\tilde{\phi}^{k}$ to satisfy (6) is that their corresponding symbols $a^{k}(z)$ and $\tilde{a}^{k}(z)$ satisfy

$$
a^{k}(z) \tilde{a}^{k}\left(z^{-1}\right)+a^{k}(-z) \tilde{a}^{k}\left(-z^{-1}\right)=4, \quad k \geq 0 .
$$

Thus, the construction of $\tilde{\phi}^{k}$ starts from finding the symbol $\tilde{a}^{k}(z)$ satisfying (9).

From Sect. 2, $a^{k}(z)$ contains the factor $b^{k}(z)$, which is the symbol of the exponential Bspline scheme generating $E P_{\Gamma}$. Indeed, we want $\tilde{a}^{k}(z)$ to have the same factor $b^{k}(z)$. Now let $z=e^{-l \xi}, y=\sin ^{2}\left(\frac{\xi}{2}\right)$ (i.e. $y=-\frac{(1-z)^{2}}{4 z}$ ). Thus by setting

$$
P^{k}(y)=\frac{1}{4} a^{k}\left(e^{-l \xi}\right) \overline{b^{k}\left(e^{-l \xi}\right)}
$$

the problem of finding the desired $\tilde{a}^{k}(z)$ satisfying (9) reduces to finding $Q^{k}(y)$ (with $\left.\tilde{a}^{k}\left(e^{-l \xi}\right)=b^{k}\left(e^{-l \xi}\right) Q^{k}(y)\right)$ satisfying the Bezout equation,

$$
P^{k}(y) Q^{k}(y)+P^{k}(1-y) Q^{k}(1-y)=1, \quad y \in[0,1], k \geq 0 .
$$

In this way, by finding the solution $Q^{k}(y)$ to (10), the symbol $\tilde{a}^{k}(z)$ satisfying (9) can be found. For this purpose, we need to show that $P^{k}(y)$ and $P^{k}(1-y)$ have no common roots on $[0,1]$. In fact, from (5), $b^{k}(z)$ is symmetric about $z^{0}$ and thus satisfies $b^{k}(z)=b^{k}\left(z^{-1}\right)$. Besides, since $a^{k}(z)$ is the $k$ level symbol of the pseudo-spline scheme generating and reproducing the same space $E P_{\Gamma}$, from [13], $a^{k}(z)$ has the same symmetry as $b^{k}(z)$ and satisfies $a^{k}(z)=a^{k}\left(z^{-1}\right)$. In this way, $P^{k}(y)$ and $P^{k}(1-y)$ have no common roots on $[0,1]$ if $a^{k}(z) b^{k}\left(z^{-1}\right)=a^{k}(z) b^{k}(z)$ has no symmetric zeros on $\mathbb{C} \backslash\{0\}$. Since $b^{k}(z)$ is the symbol of the exponential B-spline scheme generating $E P_{\Gamma}$ and $a^{k}(z)$ is the symbol of the interpolatory exponential pseudo-spline reproducing $E P_{\Gamma}$, both of these two kinds of refinable functions are linearly independent and the symbols $a^{k}(z), b^{k}(z)$ have no symmetric zeros on $\mathbb{C} \backslash\{0\}[3,12]$. Thus, $a^{k}(z) b^{k}(z)$ also has no symmetric zeros on $\mathbb{C} \backslash\{0\}$.

With the set $\Gamma$ in (3), we see that $\sharp E P_{\Gamma}=2 m$ and thus $P^{k}(y)$ is of degree $3 m-1$. Then, by solving (10), we can find the unique solution $Q^{k}(y)$ of degree $3 m-2$ and thus the corresponding $\tilde{a}^{k}(z)$ satisfying (9) can be found. As a result, it can be found that the limit stationary counterpart of $Q^{k}(y)$ is just $P_{2}(y)$ (dependent on $N \in \mathbb{Z}_{+}$and $\omega \in \mathbb{R}$ ) in Sect. 6 in [3] with $N=m, \omega=0$. Thus, the limit stationary counterparts of $a^{k}(z)$ and $\tilde{a}^{k}(z)$ are the two symbols found with $N=m, \omega=0$ in [3].

In fact, in the stationary case, the solutions to (9) can be found in several ways. One of them is the method of construction by cosets $(C B C)$ [20,21], which gives the minimal support of the dual refinable functions for a given order of sum rules [21]. Besides, Dong and Shen [4] gave a similar way to derive the symbols of the dual refinable functions of the pseudo-splines. Now based on the work by Dong and Shen [4], we give the following result on the solution to (9).

Theorem 2 With the solution $Q^{k}(y)$ of degree $3 m-2$ to (10), there exists the following solution $\tilde{a}^{k}(z)$ to (9):

$$
\tilde{a}^{k}(z)=a^{k}(z)\left(3-a^{k}(z)\right),
$$

which satisfies $\tilde{a}^{k}\left(e^{-l \xi}\right)=b^{k}\left(e^{-l \xi}\right) Q^{k}(y)$ with $b^{k}(z)$ as in (5) and $z=e^{-l \xi}$. 
Proof We first show that $\tilde{a}^{k}(z)$ in (11) satisfies (9). In fact, the exponential pseudo-spline with the symbol $a^{k}(z)$ is an interpolatory refinable function and $a^{k}(z)$ can actually be rewritten as

$$
a^{k}(z)=a_{0}^{k}(z)+a_{1}^{k}(z):=1+\sum_{j \in \mathbb{Z}} a_{2 j+1}^{k} z^{2 j+1} .
$$

Thus since $a^{k}(z)=a^{k}\left(z^{-1}\right)$, it can be computed that $\tilde{a}^{k}(z)$ in (11) and $a^{k}(z)$ satisfy

$$
\begin{aligned}
& a^{k}(z) \tilde{a}^{k}\left(z^{-1}\right)+a^{k}(-z) \tilde{a}^{k}\left(-z^{-1}\right) \\
& \quad=a^{k}(z)\left[a^{k}\left(z^{-1}\right)\left(3-a^{k}\left(z^{-1}\right)\right)\right]+a^{k}(-z)\left[a^{k}\left(-z^{-1}\right)\left(3-a^{k}\left(-z^{-1}\right)\right)\right] \\
& \quad=\left(1+a_{1}^{k}(z)\right)\left[3\left(1+a_{1}^{k}(z)\right)-\left(1+a_{1}^{k}(z)\right)^{2}\right]+\left(1-a_{1}^{k}(z)\right)\left[3\left(1-a_{1}^{k}(z)\right)-\left(1-a_{1}^{k}(z)\right)^{2}\right] \\
& \quad=4 .
\end{aligned}
$$

Therefore, $\tilde{a}^{k}(z)$ in (11) satisfies (9). Besides, with $y=\sin ^{2}\left(\frac{\xi}{2}\right)$ and $z=e^{-l \xi}, \tilde{a}^{k}\left(e^{-l \xi}\right)$ is of degree $4 m-2$ in terms of $y$, since $a^{k}\left(e^{-l \xi}\right)$ is of degree $2 m-1$ in terms of $y$. Then since $b^{k}\left(e^{-l \xi}\right)$ is of degree $m$ in terms of $y, \frac{\tilde{a}^{k}\left(e^{-l \xi}\right)}{b^{k}\left(e^{-l \xi}\right)}$ is of degree $3 m-2$ in terms of $y$ and we denote it by $R^{k}(y)$. Thus $R^{k}(y)=Q^{k}(y)$, since the solution of degree $3 m-2$ to (10) is unique. Therefore, $\tilde{a}^{k}(z)$ in (11) satisfies $\tilde{a}^{k}\left(e^{-l \xi}\right)=b^{k}\left(e^{-l \xi}\right) Q^{k}(y)$.

Now with the dual symbol $\tilde{a}^{k}(z)$ found, the only candidates for the refinable and dual refinable functions can be defined through the Fourier transforms as

$$
\hat{\phi}^{k}(\xi)=\prod_{n=0}^{\infty} \frac{1}{2} a^{k+n}\left(e^{-l \xi 2^{-n-1}}\right), \quad \hat{\tilde{\phi}}^{k}(\xi)=\prod_{n=0}^{\infty} \frac{1}{2} \tilde{a}^{k+n}\left(e^{-l \xi 2^{-n-1}}\right) .
$$

Denote the limit stationary counterparts of $a^{k}(z)$ and $\tilde{a}^{k}(z)$ by $a(z)$ and $\tilde{a}(z)$ and their associated refinable functions (defined as in (12)) by $\phi$ and $\tilde{\phi}$. Then $\phi$ and $\tilde{\phi}$ are the refinable functions in the Coifman biorthogonal wavelet systems obtained by the masks of the D-D interpolatory subdivision, i.e. the refinable functions in [3] with $N=m, \omega=0$ (see also the refinable functions in Sect. 3 in [4]). According to [3, 4], the regularity and stability of $\phi$ and $\tilde{\phi}$ are known. In fact, $\phi$ and $\tilde{\phi}$ are just the limit stationary counterparts of $\phi^{k}$ and $\tilde{\phi}^{k}$ and, by Theorem 3 in [22], $\phi^{k}$ and $\tilde{\phi}^{k}$ have the same regularity as $\phi$ and $\tilde{\phi}$, respectively.

Now we show that $\tilde{\phi}^{k}$ is indeed a dual refinable function of $\phi^{k}$. Let the symbols $a^{k}(z)$, $\tilde{a}^{k}(z)$ and their stationary counterparts $a(z), \tilde{a}(z)$ be written as

$$
a^{k}(z)=\prod_{j=0}^{n}\left(\frac{1+e^{\theta_{j} 2^{-k-1}} z}{1+e^{\theta_{j} 2^{-k-1}}}\right)^{\tau_{j}} c^{k}(z), \quad \tilde{a}^{k}(z)=\prod_{j=0}^{\tilde{n}}\left(\frac{1+e^{\tilde{\theta}_{j} 2^{-k-1}} z}{1+e^{\tilde{\theta}_{j} 2^{-k-1}}}\right)^{\tilde{\tau}_{j}} \tilde{c}^{k}(z),
$$

where

$$
\left(\theta_{j}, \tau_{j}\right),\left(\tilde{\theta}_{j}, \tilde{\tau}_{j}\right) \in \Gamma, \quad l=\sum_{j=0}^{n} \tau_{j}, \quad \tilde{l}=\sum_{j=0}^{\tilde{n}} \tilde{\tau}_{j}
$$


and

$$
a(z)=\left(\frac{1+z}{2}\right)^{l} c(z), \quad \tilde{a}(z)=\left(\frac{1+z}{2}\right)^{\tilde{l}} \tilde{c}(z) .
$$

It can be seen that $l, \tilde{l} \leq 2 \mathrm{~m}$. Now we suppose that there exist $j, \tilde{j} \in \mathbb{Z}_{+}$such that $\left\|\left(\frac{1}{2} S_{\boldsymbol{c}}\right)^{j}\right\|_{\infty}, \|\left(\frac{1}{2} S_{\tilde{\mathbf{c}}} \tilde{j}^{j} \|_{\infty}<1\right.$, where $S_{\boldsymbol{c}}$ and $S_{\tilde{\boldsymbol{c}}}$ are the schemes whose symbols are $c(z)$ and $\tilde{c}(z)$, respectively, and the norm $\left\|\left(\frac{1}{2} S_{c}\right)^{j}\right\|_{\infty},\left\|\left(\frac{1}{2} S_{\tilde{c}}\right)^{\tilde{j}}\right\|_{\infty}$ are defined through the definition

$$
\left\|S_{\boldsymbol{q}}^{L}\right\|_{\infty}=\max \left\{\sum_{j \in \mathbb{Z}}\left|q_{i-2^{k} j}^{[L]}\right|: 0 \leq i<2^{L}\right\}, \quad \boldsymbol{q} \in l_{0}(\mathbb{Z}), L \in \mathbb{Z}_{+} .
$$

This implies that $S_{\boldsymbol{a}} \in C^{l-1}, S_{\tilde{\boldsymbol{a}}} \in C^{\tilde{l}-1}[23]$.

Now define

$$
\begin{aligned}
& C_{k, j}=\sup _{n \geq k} \max _{\xi}\left|\prod_{l=1}^{j} \frac{1}{2} c^{n+l-1}\left(e^{-l \xi 2^{-l}}\right)\right|^{1 / j}, \quad \tilde{C}_{k, \tilde{j}}=\sup _{n \geq k} \max _{\xi}\left|\prod_{l=1}^{\tilde{j}} \frac{1}{2} \tilde{c}^{n+l-1}\left(e^{-l \xi 2^{-l}}\right)\right|^{1 / \tilde{j}}, \\
& C_{j}=\max _{\xi}\left|\prod_{l=1}^{j} \frac{1}{2} c\left(e^{-l \xi 2^{-l}}\right)\right|^{1 / j}, \quad \tilde{C}_{\tilde{j}}=\max _{\xi}\left|\prod_{l=1}^{\tilde{j}} \frac{1}{2} \tilde{c}\left(e^{-l \xi 2^{-l}}\right)\right|^{1 / \tilde{j}} \cdot
\end{aligned}
$$

Then we have the following result showing that $\tilde{\phi}^{k}$ is indeed a dual refinable function of $\phi^{k}$.

Theorem 3 For the refinable functions $\phi$ and $\tilde{\phi}$ with the symbols $a(z)$ and $\tilde{a}(z)$ in (14), if $\left\|\left(\frac{1}{2} S_{c}\right)^{j}\right\|_{\infty},\left\|\left(\frac{1}{2} S_{\tilde{c}}\right)^{\tilde{j}}\right\|_{\infty}<1$ for some $j, \tilde{j} \in \mathbb{Z}_{+}$, then, for $l, \tilde{l}$ satisfying $l+\tilde{l} \geq 3$, the refinable functions $\phi^{k}$ and $\tilde{\phi}^{k}$ satisfy

$$
\left\langle\phi^{k}, \tilde{\phi}^{k}(\cdot-n)\right\rangle=\delta_{n, 0}, \quad k \geq 0
$$

Proof In fact, the masks $\boldsymbol{a}, \tilde{\boldsymbol{a}}$ corresponding to the symbols $a(z), \tilde{a}(z)$ in $(14)$ are in $l_{0}(\mathbb{Z})$ and the assumptions imply that $S_{\boldsymbol{a}} \in C^{l-1}$ and $S_{\tilde{\boldsymbol{a}}} \in C^{\tilde{l}-1}$. Therefore, the associated refinable functions $\phi \in C^{l-1}$ and $\tilde{\phi} \in C^{\tilde{l}-1}$ are compactly supported. Thus $\phi, \tilde{\phi} \in L^{2}(\mathbb{R})$. Besides, similar to the proof of Theorem 5.1 in [3] (the proof of Theorem 5.3 in [24]), it can be deduced that

$$
C_{j} \leq 2\left(\left\|\left(\frac{1}{2} S_{c}\right)^{j}\right\|_{\infty}\right)^{1 / j}, \quad \tilde{C}_{\tilde{j}} \leq 2\left(\left\|\left(\frac{1}{2} S_{\tilde{c}}\right)^{\tilde{j}}\right\|_{\infty}\right)^{1 / \tilde{j}} .
$$

Thus, $C_{j} \tilde{C}_{\tilde{j}}<4$ and for $l, \tilde{l}$ satisfying $l+\tilde{l} \geq 3, C_{j} \tilde{C}_{\tilde{j}}<2^{l+\tilde{l}-1}$.

Since $\boldsymbol{a}^{k}, \boldsymbol{a} \in l_{0}(\mathbb{Z})$, according to the convergence rate result (Theorem 2.7) in [17], we have

$$
\left\|\boldsymbol{a}^{k}-\boldsymbol{a}\right\|_{\infty}=O\left(2^{-k}\right), \quad\left\|\boldsymbol{a}^{k}-\boldsymbol{a}\right\|_{1}=O\left(2^{-k}\right)
$$

then $a^{k}(z)$ converges to $a(z)$ uniformly on $|z|=1$ as $k$ tends to $\infty$. This also holds for $\tilde{a}^{k}(z)$ and $\tilde{a}(z)$. Then it can be deduced that there exists $K_{1}>0$ such that, for $k>K_{1}, C_{k, j} \tilde{C}_{k, j}<$ $2^{l+\tilde{l}-1}$. 
Now define

$$
\begin{aligned}
& u_{k, p}(\xi):=\left[\prod_{l=1}^{p} \frac{1}{2} a^{k+l-1}\left(e^{-l \xi 2^{-l}}\right)\right] \chi_{[-\pi, \pi]}\left(\xi 2^{-p}\right), \\
& \tilde{u}_{k, p}(\xi):=\left[\prod_{l=1}^{p} \frac{1}{2} \tilde{a}^{k+l-1}\left(e^{-l \xi 2^{-l}}\right)\right] \chi_{[-\pi, \pi]}\left(\xi 2^{-p}\right) .
\end{aligned}
$$

Then similar to the proof of Lemma 3.3 in [12], for sufficiently large $k$ (say $k>K_{2}$ for some $\left.K_{2}>0\right)$,

$$
\left|u_{k, p}(\xi) \tilde{u}_{k, p}(\xi)\right| \leq c_{0}(1+|\xi|)^{-l-\tilde{l}+\log _{2}\left(C_{k, j} \tilde{C}_{k, \tilde{j}}\right)}
$$

where $c_{0}$ is independent of $p$.

Since $\boldsymbol{a}^{k}, \tilde{\boldsymbol{a}}^{k} \in l_{0}(\mathbb{Z})$ and the associated refinable functions $\phi^{k}, \tilde{\phi}^{k}$ have the same regularity as $\phi$ and $\tilde{\phi}$, the refinable functions $\phi^{k}, \tilde{\phi}^{k}$ are at least $C^{0}$ and compactly supported and thus $\phi^{k}, \tilde{\phi}^{k} \in L^{2}(\mathbb{R})$. Then, for $k>K:=\max \left\{K_{1}, K_{2}\right\}, u_{k, p} \overline{\tilde{u}_{k, p}} \rightarrow \hat{\phi}^{k} \overline{\tilde{\tilde{\phi}}}^{k}$ in $L^{1}(\mathbb{R})$ as $p \rightarrow \infty$ by the Lebesgue-dominated convergence theorem. Together with Placherel's theorem and the fact that

$$
\int_{\mathbb{R}} u_{k, p}(\xi) \overline{\tilde{u}_{k, p}}(\xi) e^{\imath n \xi} d x=\int_{\mathbb{R}} u_{k, 1}(\xi) \overline{\tilde{u}_{k, 1}}(\xi) e^{\imath n \xi} d x=2 \pi \delta_{n, 0},
$$

it can be seen that (16) holds for $k>K$.

For the case $k \leq K$, the desired result can be derived by an inductive argument based on the non-stationary refinement equation (see Proposition 3.5 in [12]).

Remark 1 When $\sharp E P_{\Gamma}=2$, the corresponding exponential pseudo-spline cannot be included in Theorem 3. Yet, this exponential pseudo-spline is actually the exponential Bspline of order 2 and the corresponding construction and analysis of the dual refinable function can be found in $[11,12]$.

\subsection{Stability}

We first give a result on the convergence rate of $\phi^{k}$ and $\tilde{\phi}^{k}$ (see also $[11,12]$ for another proof).

Lemma 4 For the refinable functions $\phi^{k}$ and $\tilde{\phi}^{k}$, we have $\left\|\phi^{k}-\phi\right\|_{L^{2}(\mathbb{R})}=O\left(2^{-k}\right)$ and $\| \tilde{\phi}^{k}-$ $\tilde{\phi} \|_{L^{2}(\mathbb{R})}=O\left(2^{-k}\right)$.

Proof According to the proof of Lemma 15 in [25] and the result that $\left\|\boldsymbol{a}^{k}-\boldsymbol{a}\right\|_{\infty}=O\left(2^{-k}\right)$, we have

$$
\left\|\phi^{k}-\phi\right\|_{\infty} \leq c_{1} \sum_{j=0}^{\infty}\left\|S_{\boldsymbol{a}^{k+j}}-S_{\boldsymbol{a}}\right\|_{\infty} \leq c_{1} \sum_{j=0}^{\infty} 2^{-k-j}=c_{2} 2^{-k}
$$

where $c_{1}$ is a positive constant independent of $k, c_{2}=2 c_{1}$ and the norm $\left\|S_{\boldsymbol{a}^{k+j}}-S_{\boldsymbol{a}}\right\|_{\infty}$ is defined through (15) with $L=1$ and $\boldsymbol{q}=\boldsymbol{a}^{k+j}-\boldsymbol{a}$. As for $\tilde{\phi}^{k}$, from (9), $\tilde{\boldsymbol{a}}^{k} \in l_{0}(\mathbb{Z})$. Then, in a similar way, we can obtain the same result for $\tilde{\phi}^{k}$. 
With the obtained dual refinable functions, we need to verify their stability. In fact, this can be done following the lines of [12] (see Sect. 4). Here, for the purpose of illustration, we show that $\tilde{\phi}^{k}$ is stable for each fixed $k \geq 0$.

Lemma 5 There exist constants $\tilde{A}, \tilde{B}>0$ such that

$$
\tilde{A} \leq\left[\hat{\tilde{\phi}}^{k}, \hat{\tilde{\phi}}^{k}\right] \leq \tilde{B}, \quad k \geq 0 .
$$

Proof Note that, for each $k \geq 0, \tilde{\phi}^{k} \in L^{2}(\mathbb{R})$ and is compactly supported. Thus, there exists $\tilde{B}^{k}$ such that

$$
\left[\hat{\tilde{\phi}}^{k}, \hat{\tilde{\phi}}^{k}\right] \leq \tilde{B}^{k} .
$$

Since $\left\langle\phi^{k}, \tilde{\phi}^{k}(\cdot-n)\right\rangle=\delta_{n, 0}$, we have $\left[\hat{\phi}^{k}, \hat{\tilde{\phi}}^{k}\right]=1$. Then, by the Cauchy-Schwartz inequality, we can obtain

$$
1 \leq\left[\hat{\phi}^{k}, \hat{\phi}^{k}\right]\left[\hat{\tilde{\phi}}^{k}, \hat{\tilde{\phi}}^{k}\right]
$$

Set $\tilde{A}^{k}=\left(\tilde{B}^{k}\right)^{-1}$, then $\tilde{A}^{k} \leq\left[\hat{\tilde{\phi}}^{k}, \hat{\tilde{\phi}}^{k}\right] \leq \tilde{B}^{k}$.

Now rewrite $\left[\hat{\tilde{\phi}}^{k}, \hat{\tilde{\phi}}^{k}\right]$ in the form

$$
\left[\hat{\tilde{\phi}}^{k}, \hat{\tilde{\phi}}^{k}\right]=\sum_{n \in \mathbb{Z}} \tilde{c}_{n}^{k} e^{l n \xi}, \quad \tilde{c}_{n}^{k}=\left\langle\tilde{\phi}^{k}, \tilde{\phi}^{k}(\cdot-n)\right\rangle \text {. }
$$

From Lemma 4 , it can be shown that, for each $n, \tilde{c}_{n}^{k}$ converges to $\tilde{c}_{n}=\langle\tilde{\phi}, \tilde{\phi}(\cdot-n)\rangle$ as $k$ tends to $\infty$. Then we have

$$
\left[\hat{\tilde{\phi}}^{k}, \hat{\tilde{\phi}}^{k}\right](\xi) \rightarrow[\hat{\tilde{\phi}}, \hat{\tilde{\phi}}](\xi), \quad k \rightarrow \infty .
$$

In this way, we see that $\tilde{A}^{k}$ and $\tilde{B}^{k}$ converge to $\tilde{A}_{\tilde{\phi}}$ and $\tilde{B}_{\tilde{\phi}}$, i.e. the lower and upper Riesz bounds of $\tilde{\phi}$. Thus, there exist $\epsilon>0$ and $\tilde{K}$ such that, for $k>\tilde{K}, \tilde{A}^{k}>\tilde{A}_{\tilde{\phi}}-\epsilon>0$ and $\tilde{B}^{k}<$ $\tilde{B}_{\tilde{\phi}}+\epsilon$. Let

$$
\tilde{A}=\min \left\{\tilde{A}^{1}, \tilde{A}^{2}, \ldots, \tilde{A}^{\tilde{K}}, \tilde{A}_{\tilde{\phi}}-\epsilon\right\}, \quad \tilde{B}=\max \left\{\tilde{B}^{1}, \tilde{B}^{2}, \ldots, \tilde{B}^{\tilde{K}}, \tilde{B}_{\tilde{\phi}}+\epsilon\right\}
$$

then we have $\tilde{A} \leq\left[\hat{\tilde{\phi}}^{k}, \hat{\tilde{\phi}}^{k}\right] \leq \tilde{B}$.

\section{The generalized Coifman biorthogonal wavelets}

With the refinable function $\phi^{k}$ and its dual refinable function $\tilde{\phi}^{k}$ obtained in Sect. 3, in this section, we obtain and analyze the corresponding non-stationary biorthogonal wavelets. Then we present an example.

\subsection{The non-stationary biorthogonal wavelets}

Based on the refinable functions $\phi^{k}$ and $\tilde{\phi}^{k}$, according to (7), the corresponding biorthogonal wavelets can be defined by

$$
\hat{\psi}^{k}(\xi)=g(\xi / 2) \hat{\phi}^{k+1}(\xi / 2), \quad \hat{\tilde{\psi}}^{k}(\xi)=\tilde{g}(\xi / 2) \hat{\tilde{\phi}}^{k+1}(\xi / 2),
$$


where

$$
g(\xi)=\frac{1}{2} e^{-l \xi} \overline{\tilde{a}^{k}\left(e^{-l(\xi+\pi)}\right)}, \quad \tilde{g}(\xi)=\frac{1}{2} e^{-l \xi} \overline{a^{k}\left(e^{-l(\xi+\pi)}\right)}
$$

From (17), it can be seen that the limit stationary counterparts of $\psi^{k}$ and $\tilde{\psi}^{k}$ are the wavelet functions obtained in [3] with $N=m, \omega=0$.

Remark 2 Since the refinable functions $\phi^{k}$ and $\tilde{\phi}^{k}$ have the same regularity as their limit stationary counterparts, the wavelet functions $\psi^{k}(x)$ and $\tilde{\psi}^{k}(x)$ given in (17) also have the same regularity with their stationary counterparts.

Similar to Lemma 5, we can show that $\psi^{k}$ and $\tilde{\psi}^{k}$ are stable for each $k \geq 0$. Furthermore, following the lines of [12] (see Theorems 4.4 and 4.5), for $k_{0} \in \mathbb{N}$, we can also show the global stability of the sets $\left\{\psi_{i}^{k}: k \geq 0, i \in \mathbb{Z}\right\}$ and $\left\{\tilde{\psi}_{i}^{k}: k \geq 0, i \in \mathbb{Z}\right\}$ and that the sets $\left\{\phi_{i}^{k_{0}}\right.$ : $i \in \mathbb{Z}\} \cup\left\{\psi_{i}^{k}: k \geq k_{0}, i \in \mathbb{Z}\right\}$ and $\left\{\tilde{\phi}_{i}^{k_{0}}: i \in \mathbb{Z}\right\} \cup\left\{\tilde{\psi}_{i}^{k}: k \geq k_{0}, i \in \mathbb{Z}\right\}$ form Riesz bases of $L^{2}(\mathbb{R})$.

\subsection{Exponential vanishing moments}

From Sects. 3 and 4, the limit stationary counterparts of $\phi^{k}, \tilde{\phi}^{k}$ and $\psi^{k}, \tilde{\psi}^{k}$ form the biorthogonal Coifman wavelet system constructed using the masks of the D-D interpolatory subdivision. Recall that $\phi, \tilde{\phi}$ and $\psi, \tilde{\psi}$ form a biorthogonal Coifman wavelet system of order $L$ if the refinable function $\phi$ and the wavelets $\psi, \tilde{\psi}$ have the vanishing moments $L$ [3], i.e.,

$$
\int_{\mathbb{R}} x^{r} \phi(x) d x=\delta_{r, 0}, \quad \int_{\mathbb{R}} x^{r} \psi(x) d x=\int_{\mathbb{R}} x^{r} \tilde{\psi}(x) d x=0, \quad r=0, \ldots, L-1 .
$$

Now we consider $\psi^{k}, \tilde{\psi}^{k}$. Given the set $\Gamma$ in (3), from Sect. 2, $\left\{S_{\boldsymbol{a}^{k}}\right\}_{k \geq 0}$ reproduces $E P_{\Gamma}$. Besides, from (11), $\tilde{a}^{k}(z)$ satisfies the conditions which $a^{k}(z)$ satisfies for the reproduction of $E P_{\Gamma}$ by the scheme $\left\{S_{\boldsymbol{a}^{k}}\right\}_{k \geq 0}$. Thus $\left\{S_{\tilde{a}^{k}}\right\}_{k \geq 0}$ also reproduces $E P_{\Gamma}$. As a result, the nonstationary MRAs $\left(V^{k}\right)_{k \geq 0}$ and $\left(\tilde{V}^{k}\right)_{k \geq 0}$ based on $\phi^{k}$ and $\tilde{\phi}^{k}$ can reproduce $E P_{\Gamma}$ and thus, by (7), the wavelet functions $\psi^{k}$ and $\tilde{\psi}^{k}$ have exponential vanishing moments in the sense that (see $[11,14])$

$$
\int_{\mathbb{R}} x^{r} e^{\theta x} \psi^{k}(x) d x=\int_{\mathbb{R}} x^{r} e^{\theta x} \tilde{\psi}^{k}(x) d x=0, \quad x^{r} e^{\theta x} \in E P_{\Gamma}, k \geq 0
$$

For the refinable function $\phi^{k}$ with the symbol $a^{k}(z), k \geq 0$, recall that it is obtained as the basic limit function of the exponential pseudo-spline subdivision $\left\{S_{\boldsymbol{a}^{k}}\right\}_{k \geq 0}$ reproducing $E P_{\Gamma}$. Then we have the following results.

Theorem 6 If $E P_{\Gamma}$ contains the subset $E_{0}:=\left\{1, \ldots, x^{2 \tau-1}\right\}$ with $\tau \in \mathbb{Z}_{+}$, then $\phi^{k}$ satisfies

$$
\int_{\mathbb{R}} x^{r} \phi^{k}(x) d x=\delta_{r, 0}, \quad r=0, \ldots, 2 \tau-1
$$

Proof Denote by $D^{j} f$ the $j$ th derivative of $f \in C^{i}$ with $i, j \in \mathbb{Z}, 0 \leq j \leq i$ for simplicity. Since the subdivision $\left\{S_{\boldsymbol{a}^{k}}\right\}_{k \geq 0}$ reproduces $E P_{\Gamma},\left\{S_{\boldsymbol{a}^{k}}\right\}_{k \geq 0}$ also reproduces $E_{0}$. Then, by Theo- 
rem 1 , it can be computed that

$$
\begin{aligned}
\int_{\mathbb{R}} x^{r} \phi^{0}(x) d x & =\left({ }_{l} D\right)^{r} \hat{\phi}^{0}(0)=\left.(l D)^{r}\left(\frac{1}{2} a^{0}\left(e^{-l \xi}\right) \hat{\phi}^{1}(\xi)\right)\right|_{\xi=0}=(l D)^{r} \hat{\phi}^{1}(0) \\
& =\int_{\mathbb{R}} x^{r} \phi^{1}(x) d x .
\end{aligned}
$$

Similarly, $\int x^{r} \phi^{0}(x) d x=\int x^{r} \phi^{k}(x) d x, k \geq 0$. Note that, for the stationary counterpart $\phi(x)$ with the symbol $a(z)$, it satisfies

$$
\int_{\mathbb{R}} x^{r} \phi(x) d x=\delta_{r, 0}, \quad r=0, \ldots, 2 \tau-1
$$

Thus, we only have to show that $\lim _{k \rightarrow \infty} \int_{\mathbb{R}} x^{r} \phi^{k}(x) d x=\int_{\mathbb{R}} x^{r} \phi(x) d x$, for $r=0, \ldots, 2 \tau-1$.

Recall that $\phi^{k}$ and $\phi$ are compactly supported. We denote by $\Omega$ their compact support. Then, by the triangular inequality and the Hölder inequality, we have

$$
\begin{aligned}
\left|\int_{\mathbb{R}} x^{r}\left(\phi^{k}(x)-\phi(x)\right) d x\right| & \leq\left|\int_{\Omega} x^{r}\left(\phi^{k}(x)-\phi(x)\right) d x\right|+\left|\int_{\mathbb{R} \backslash \Omega} x^{r}\left(\phi^{k}(x)-\phi(x)\right) d x\right| \\
& =\left|\int_{\Omega} x^{r}\left(\phi^{k}(x)-\phi(x)\right) d x\right| \\
& \leq c_{3}\left\|\phi^{k}-\phi\right\|_{L^{2}(\mathbb{R})},
\end{aligned}
$$

where $r=0, \ldots, 2 \tau-1$ and $c_{3}$ is a constant independent of $n$. From Lemma $4, \lim _{k \rightarrow \infty} \| \phi^{k}-$ $\phi \|_{L^{2}(\mathbb{R})}=0$. Therefore, we can conclude that

$$
\int_{\mathbb{R}} x^{r} \phi^{0}(x) d x=\lim _{k \rightarrow \infty} \int_{\mathbb{R}} x^{r} \phi^{k}(x) d x=\int_{\mathbb{R}} x^{r} \phi(x) d x=\delta_{r, 0}, \quad r=0, \ldots, 2 \tau-1
$$

Theorem 7 If $E P_{\Gamma}$ contains $E_{1}:=\left\{e^{ \pm \theta x}, \ldots, x^{2 \tau-1} e^{ \pm \theta x}\right\}, \theta \in \mathbb{R} \cup \imath \mathbb{R}$, then $\phi^{0}$ satisfies

$$
\int_{\mathbb{R}} x^{r} e^{ \pm \theta x} \phi^{0}(x) d x=\delta_{r, 0}, \quad r=0, \ldots, 2 \tau-1
$$

Proof We show $\int_{\mathbb{R}} x^{r} e^{\theta x} \phi^{0}(x) d x=\delta_{r, 0}$ and the other case can be done similarly. In fact, similar to the proof of Theorem 6, with Theorem 1, it can be computed that

$$
\begin{aligned}
\int_{\mathbb{R}} x^{r} e^{\theta x} \phi^{0}(x) d x & =\left({ }_{l} D\right)^{r} \hat{\phi}^{0}(\imath \theta)=\left({ }_{l} D\right)^{r} \hat{\phi}^{1}(l \theta / 2)=\cdots=(l D)^{r} \hat{\phi}^{k}\left(l \theta / 2^{k}\right) \\
& =\int_{\mathbb{R}} x^{r} e^{\frac{\theta}{2^{k}}} \phi^{k}(x) d x .
\end{aligned}
$$

In this way, we only have to show $\lim _{k \rightarrow \infty} \int_{\mathbb{R}} x^{r} e^{\frac{\theta}{2^{k}} x} \phi^{k}(x) d x=\delta_{r, 0}$, with $r=0, \ldots, 2 \tau-1$.

In fact, by the triangular inequality, we have

$$
\left|\int_{\mathbb{R}} x^{r} e^{\frac{\theta}{2^{k}} x} \phi^{k}(x) d x\right| \leq\left|\int_{\mathbb{R}} x^{r} \phi^{k}(x)\left(1-e^{\frac{\theta}{2^{k} x}}\right) d x\right|+\left|\int_{\mathbb{R}} x^{r} \phi^{k}(x) d x\right| .
$$


It can be seen that $\lim _{k \rightarrow \infty}\left|\int_{\mathbb{R}} x^{r} \phi^{k}(x)\left(1-e^{\frac{\theta}{2^{k}} x}\right) d x\right|=0$. Then from Theorem 6, $\lim _{k \rightarrow \infty} \int_{\mathbb{R}} x^{r} \phi^{k}(x) d x=\delta_{r, 0}$, for $r=0, \ldots, 2 \tau_{r}-1$. Therefore, we see that

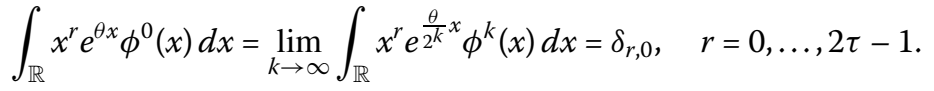

Since $\left\{S_{\tilde{\boldsymbol{a}}^{k}}\right\}_{k \geq 0}$ reproduces $E P_{\Gamma}, \tilde{\phi}^{k}$ can also be shown to have the same exponential vanishing moments as $\phi^{k}$ does. In this way, both the refinable functions and the wavelet functions have the exponential vanishing moments and their limit stationary counterparts form the Coifman biorthogonal wavelet system of order $2 m$.

\subsection{An example}

We take the exponential pseudo-spline with the symbol $a^{k}(z)$ as an example, where

$$
a^{k}(z)=-\frac{1}{8 v^{k+1}\left(v^{k+1}+1\right)}\left(z^{-3}+z^{3}\right)+\frac{\left(2 v^{k+1}+1\right)^{2}}{8 v^{k+1}\left(v^{k+1}+1\right)}\left(z+z^{-1}\right)+1
$$

It is known that the limit stationary counterpart is just the pseudo-spline of type II with order $(2,1)$. The corresponding exponential pseudo-spline subdivision is the interpolatory scheme reproducing $E^{\prime}=\left\{1, x, e^{ \pm t x}\right\}$ (see also [26]).

By Theorem 2, the corresponding dual symbols can be derived and the refinable and wavelet functions can then be obtained. The figures of the refinable and wavelet functions with different values of $v^{0}$ are shown in Fig. 1.
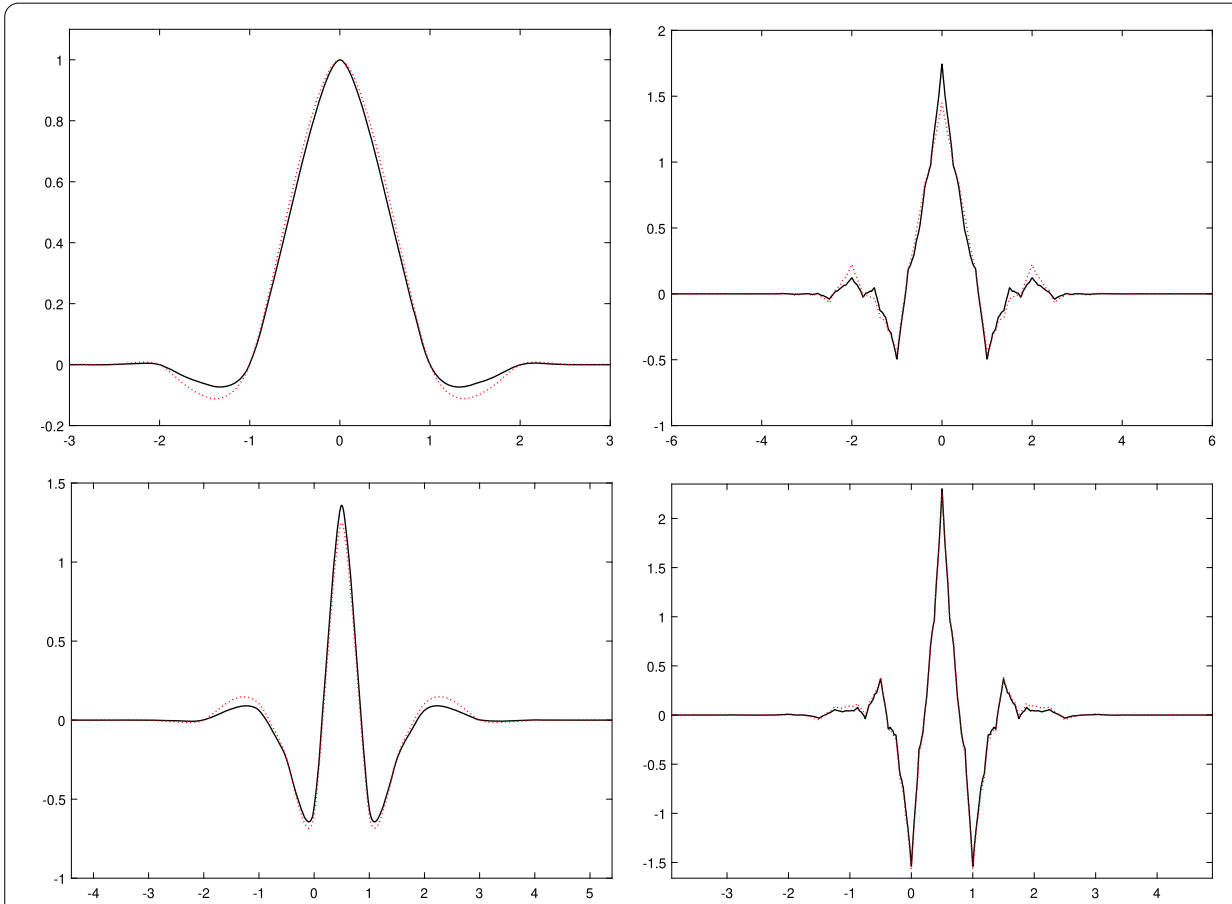

Figure 1 The refinable function with the symbol in (18), the dual refinable function and the corresponding wavelet functions at scale 0 with $v^{0}=0$ (dashed line) and $v^{0}=1$ (solid line). From left to right and top to bottom: $\phi^{0}, \tilde{\phi}^{0}, \psi^{0}$ and $\tilde{\psi}^{0}$ 


\section{Conclusion}

This paper presented a family of non-stationary biorthogonal wavelets. This family of biorthogonal wavelets is based on a subclass of the exponential pseudo-splines, which actually consists of interpolatory cardinal functions. For this family of biorthogonal wavelets, we showed the stability of the refinable and wavelet functions. Besides, we showed that both of the refinable and wavelet functions have the exponential vanishing moments and can be seen as a non-stationary generalization of the Coifman biorthogonal wavelet systems constructed using the masks of the D-D interpolatory subdivision. Another advantage of these non-stationary biorthogonal wavelets is that they offer a degree of freedom, which makes it possible to tune these new biorthogonal wavelets to certain classes of signals.

Taking the above points into consideration, we can envisage the use of these new biorthogonal wavelets in applications like the approximation and sparse representation of the band limited signals, since such signals usually have exponential trends. Based on this, future work may focus on such applications of these new biorthogonal wavelets.

\section{Acknowledgements}

The authors thank all the reviewers for their careful reading and valuable comments.

\section{Funding}

This work is supported by the National Science Foundation for Young Scientists of China (Grant No. 61801389).

Availability of data and materials

The datasets used during the current study are available from the corresponding author on reasonable request.

Competing interests

The authors declare that they have no competing interests.

Authors' contributions

All authors contributed to this work equally. All authors read and approved the final manuscript.

\section{Publisher's Note}

Springer Nature remains neutral with regard to jurisdictional claims in published maps and institutional affiliations.

Received: 24 May 2019 Accepted: 29 October 2019 Published online: 08 November 2019

\section{References}

1. Cohen, A., Daubechies, l., Feauveau, J.-C.: Biorthogonal bases of compactly supported wavelets. Commun. Pure Appl. Math. 45, 485-560 (1992)

2. Wei, D., Tian, J., Wells, R., Burrus, C.S.: A new class of biorthogonal wavelet systems for image transform coding. IEEE Trans. Image Process. 7, 1000-1013 (1998)

3. Kim, H., Kim, R., Lee, Y., Yoon, J.: Quasi-interpolatory refinable functions and construction of biorthogonal wavelet systems. Adv. Comput. Math. 33, 255-283 (2010)

4. Dong, B., Shen, Z.: Construction of biorthogonal wavelets from pseudo-splines. J. Approx. Theory 138, 211-231 (2006)

5. Zhou, J., Zheng, H.: Biorthogonal wavelets and tight framelets from smoothed pseudo splines. J. Inequal. Appl. 2017, Article ID 166 (2017)

6. Choi, S., Lee, B., Lee, Y., Yoon, J.: Stationary subdivision schemes reproducing polynomials. Comput. Aided Geom. Des. 23, 351-360 (2006)

7. Daubechies, I., Han, B., Ron, A., Shen, Z.: Framelets: MRA-based constructions of wavelet frames. Appl. Comput. Harmon. Anal. 14, 1-46 (2003)

8. Dong, B., Shen, Z.: Pseudo-splines, wavelets and framelets. Appl. Comput. Harmon. Anal. 22, $78-104$ (2007)

9. Dyn, N., Hormann, K., Sabin, M., Shen, Z.: Polynomial reproduction by symmetric subdivision schemes. J. Approx. Theory 155, 28-42 (2008)

10. Chuang, Z., Yang, J.: A class of generalized pseudo-splines. J. Inequal. Appl. 2014, Article ID 359 (2014)

11. Vonesch, C., Blu, T., Unser, M.: Generalized Daubechies wavelet faimilies. IEEE Trans. Signal Process. 55, 4415-4429 (2007)

12. Lee, Y., Yoon, J.: Analysis of compactly supported nonstationary biorthogonal wavelet systems based on exponential B-splines. Abstr. Appl. Anal. 2011, Article ID 593436 (2011)

13. Conti, C., Gemignani, L., Romani, L.: Exponential pseudo-splines: looking beyond exponential B-splines. J. Math. Anal. Appl. 439, 32-56 (2016) 
14. Dyn, N., Kounchev, O., Levin, D., Render, H.: Regularity of generalized Daubechies wavelets reproducing exponential polynomials with real-valued parameters. Appl. Comput. Harmon. Anal. 37, 288-306 (2014)

15. Dyn, N., Levin, D., Yoon, J.: Analysis of univariate non-stationary subdivision schemes with application to Gaussian-based interpolation schemes. SIAM J. Math. Anal. 39, 470-488 (2007)

16. Charina, M., Conti, C., Romani, L.: Reoriduction of exponential polynomials by multivariate non-stationary subdivision schemes with a general dilation matrix. Numer. Math. 127, 223-254 (2014)

17. Dyn, N., Levin, D., Luzzatto, A.: Exponentials reproducing subdivision schemes. Found. Comput. Math. 3, 187-206 (2003)

18. der Boor, C., DeVore, R.A., Ron, A.: On the construction of multivariate (pre)wavelets. Constr. Approx. 9, $123-166$ (1993)

19. Jia, R., Wang, J.: Stability and linear independence associated with wavelet decompositions. Proc. Am. Math. Soc. 117 1115-1124 (1993)

20. Han, B.: Analysis and construction of optimal multivariate biorthogonal wavelets with compact support. SIAM J. Math. Anal. 31, 274-304 (1999)

21. Han, B.: Framelets and Wavelets: Algorithms, Analysis and Applications. Springer, Cham (2017)

22. Chrina, M., Conti, C., Guglielmi, N., Protasov, V.: Regularity of non-stationary subdivision: a matrix approach. Numer. Math. 135, 639-678 (2017)

23. Dyn, N., Levin, D.: Subdivision schemes in geometric modelling. Acta Numer. 11, 73-144 (2002)

24. Kim, H., Kim, R., Lee, Y., Yoon, J.: Quasi-interpolatory refinable functions and construction of biorthogonal wavelet systems. Research Report Series 06-13 of KAIST, 2006

25. Dyn, N., Levin, D.: Analysis of asymptotically equivalent binary subdivision schemes. J. Math. Anal. Appl. 193, 594-621 (1995)

26. Beccari, C., Casciola, G., Romani, L.: A non-stationary uniform tension controlled interpolating 4-point scheme reproducing conics. Comput. Aided Geom. Des. 24, 1-9 (2007)

\section{Submit your manuscript to a SpringerOpen ${ }^{\circ}$ journal and benefit from:}

- Convenient online submission

- Rigorous peer review

- Open access: articles freely available online

- High visibility within the field

- Retaining the copyright to your article

Submit your next manuscript at $>$ springeropen.com 